\title{
An Examination in IFS and IVIFS based on Various Distance Measure
}

\author{
A. Manonmani, M. Suganya
}

\begin{abstract}
There are many distance measure in Fuzzy such as Hamming, Normalized Hamming, Euclidean, Normalized Euclidean, Geometric, Normalized Geometric, Hausdroff's etc... we discussed, the comparison between IFS and IVIFS based on various distance measure is explored. IFS is converted into IVIFS in various steps. The distance between IFS and IVIFS are also compared.

Index Terms: Hamming distance measure, Normalized Hamming distance measure, Euclidean distance measure, Normalized Euclidean distance measure, Geometric distance measure, Normalized Geometric distance measure, Hausdroff's distance measure.
\end{abstract}

\section{INTRODUCTION}

A fuzzy set is a pair where is a set and a membership function. Fuzzy set is a mathematical model of vague qualitative or quantitative data, frequently generated by means of the natural language. Ther are many types of fuzzy sets. Particularly here we have taken Intuitionistic fuzzy sets. Intuitionistic fuzzy sets are sets which are represented by its membership and non-membership function. And also here we have converted Intuitionistic fuzzy sets into Interval Valued Intuitionistic fuzzy sets. Since Interval Valued Intuitionistic fuzzy sets is also represented by its membership and non-membership function which lies within an interval. After converting IFS into IVIFS both the sets are compared using various distance measure like Hamming, Normalized Hamming, Euclidean, Normalized Euclidean, Hausdroff, Geometric, Normalized Geometric distance measures.

A Numerical example is also given to explain the above mentioned concept and the distance measures are ranked separately both for IFS and IVIFS and the best distance measure and best fuzzy set among the IFS and IVIFS is detected.

In Chapter 2 we have discussed the different types of distance measure both in IFS and IVIFS.Chapter 3 explains the various steps in conversion of IFS into IVIFS. In Chapter 4 an numwerical example is proposed in which IFS and IVIFS is compared based on various distance measures.The Conculsion is explained in chapter 5 .

\section{DISTANCE MEASURES IN FUZZY SETS}

2.1 Distance measure formulae in IFS:

2.1.1 The Hamming distance measure

Revised Manuscript Received on November 11, 2019.

A. Manonmani, Assistant Professor, LRG Govt Arts College for Women, Tirupur, Tamilnadu, India.

M. Suganya, Assistant Professor, St.Joseph's College for Women, Kangeyam Road, Tirupur, Tamilnadu, India
$d_{H}(A, B)=\frac{1}{6} \sum_{i=1}^{n}\left\{\begin{array}{l}\left|u_{A}\left(x_{i}\right)-u_{B}\left(x_{i}\right)\right|+ \\ \left|v_{A}\left(x_{i}\right)-v_{B}\left(x_{i}\right)\right|+\end{array}\right\}$

2.1.2 The normalized Hamming distance measure $d_{n H}(A, B)=\frac{1}{6 n} \sum_{i=1}^{n}\left\{\begin{array}{l}\left|u_{A}\left(x_{i}\right)-u_{B}\left(x_{i}\right)\right|+ \\ \left|v_{A}\left(x_{i}\right)-v_{B}\left(x_{i}\right)\right|+\end{array}\right\}$

2.1.3 The Euclidean distance measure

$d_{E}(A, B)=\sqrt{\frac{1}{6} \sum_{i=1}^{n}\left\{\begin{array}{l}\left(u_{A}\left(x_{i}\right)-u_{B}\left(x_{i}\right)\right)^{2}+ \\ \left(v_{A}\left(x_{i}\right)-v_{B}\left(x_{i}\right)\right)^{2}\end{array}\right\}}$

2.1.4 The normalized Euclidean distance measure

$d_{n E}(A, B)=\sqrt{\frac{1}{6 n} \sum_{i=1}^{n}\left\{\begin{array}{l}\left(u_{A}\left(x_{i}\right)-u_{B}\left(x_{i}\right)\right)^{2}+ \\ \left(v_{A}\left(x_{i}\right)-v_{B}\left(x_{i}\right)\right)^{2}\end{array}\right\}}$

2.1.5 The Geometric distance measure

$$
d_{G}(A, B)=\sum_{i=1}^{n}\left\{\left(u_{A}\left(x_{i}\right)-u_{B}\left(x_{i}\right)\right)^{r}+\left(v_{A}\left(x_{i}\right)-v_{B}\left(x_{i}\right)\right)^{r}\right\}^{1 / r}
$$

2.1.6 The normalized Geometric distance measure $d_{n G}(A, B)=\frac{1}{2} \sum_{j=1}^{m} \frac{1}{6} \sum_{i=1}^{n}\left\{\left(u_{A}\left(x_{i}\right)-u_{B}\left(x_{i}\right)\right)^{r}+\left(v_{A}\left(x_{i}\right)-v_{B}\left(x_{i}\right)\right)^{r}\right\}^{1 / r}$

2.1.7 The Hausdorff distance measure

$$
d_{q}(A, B)=\frac{1}{2} \sum_{j=1}^{2} \frac{1}{3} \sum_{i=1}^{3} \max \left\{\begin{array}{l}
\left|u_{A}\left(x_{i}\right)-u_{B}\left(x_{i}\right)\right|,+ \\
\left|v_{A}\left(x_{i}\right)-v_{B}\left(x_{i}\right)\right|
\end{array}\right\}
$$

2.2 Distance measure formulae in IVIFS:

\subsubsection{The Hamming distance measure}

$$
d_{H}(A, B)=\frac{1}{6} \sum_{i=1}^{n}\left\{\begin{array}{l}
\left|u_{A}^{L}\left(x_{i}\right)-u_{B}^{L}\left(x_{i}\right)\right|+\left|p_{A}^{L}\left(x_{i}\right)-p_{B}^{L}\left(x_{i}\right)\right|+ \\
\left|v_{A}^{L}\left(x_{i}\right)-v_{B}^{L}\left(x_{i}\right)\right|+\left|u_{A}^{U}\left(x_{i}\right)-u_{B}^{U}\left(x_{i}\right)\right|+ \\
\left|p_{A}^{U}\left(x_{i}\right)-p_{B}^{U}\left(x_{i}\right)\right|+\left|v_{A}^{U}\left(x_{i}\right)-v_{B}^{U}\left(x_{i}\right)\right|
\end{array}\right\}
$$

2.2.2 The normalized Hamming distance measure

$$
d_{n H}(A, B)=\frac{1}{6 n} \sum_{i=1}^{n}\left\{\begin{array}{l}
\left|u_{A}^{L}\left(x_{i}\right)-u_{B}^{L}\left(x_{i}\right)\right|+\left|p_{A}^{L}\left(x_{i}\right)-p_{B}^{L}\left(x_{i}\right)\right|+ \\
\left|v_{A}^{L}\left(x_{i}\right)-v_{B}^{L}\left(x_{i}\right)\right|+\left|u_{A}^{U}\left(x_{i}\right)-u_{B}^{U}\left(x_{i}\right)\right|+ \\
\left|p_{A}^{U}\left(x_{i}\right)-p_{B}^{U}\left(x_{i}\right)\right|+\left|v_{A}^{U}\left(x_{i}\right)-v_{B}^{U}\left(x_{i}\right)\right|
\end{array}\right\}
$$

2.2.3 The Euclidean distance measure

$$
\left.d_{E}(A, B)=\sqrt{\frac{1}{6} \sum_{i=1}^{n}\left\{\begin{array}{l}
\left(u_{A L}\left(x_{i}\right)-u_{B L}\left(x_{i}\right)\right)^{2}+\left(\pi_{A L}\left(x_{i}\right)-\pi_{B L}\left(x_{i}\right)\right)^{2}+ \\
\left(v_{A L}\left(x_{i}\right)-v_{B L}\left(x_{i}\right)\right)^{2}+\left(u_{A U}\left(x_{i}\right)-u_{B U}\left(x_{i}\right)\right)^{2}+ \\
\left(\pi_{A U}\left(x_{i}\right)-\pi_{B U}\left(x_{i}\right)\right)^{2}+\left(v_{A U}\left(x_{i}\right)-v_{B U}\left(x_{i}\right)\right)^{2}
\end{array}\right.}\right\}
$$

2.2.4 The normalized

Euclidean distance measure 
$\left.d_{n E}(A, B)=\sqrt{\frac{1}{6 n} \sum_{i=1}^{n}\left\{\begin{array}{l}\left(u_{A}^{L}\left(x_{i}\right)-u_{B}^{L}\left(x_{i}\right)\right)^{2}+\left(p_{A}^{L}\left(x_{i}\right)-p_{B}^{L}\left(x_{i}\right)\right)^{2}+ \\ \left(v_{A}^{L}\left(x_{i}\right)-v_{B}^{L}\left(x_{i}\right)\right)^{2}+\left(u_{A}^{U}\left(x_{i}\right)-u_{B}^{U}\left(x_{i}\right)\right)^{2}+ \\ \left(p_{A}^{U}\left(x_{i}\right)-p_{B}^{U}\left(x_{i}\right)\right)^{2}+\left(v_{A}^{U}\left(x_{i}\right)-v_{B}^{U}\left(x_{i}\right)\right)^{2}\end{array}\right.}\right\}$

2.2.5 The Geometric distance measure

$d_{r}(A, B)=\sum_{i=1}^{n}\left|\left\{\begin{array}{l}\left(u_{A}^{L}\left(x_{i}\right)-u_{B}^{L}\left(x_{i}\right)\right)^{r}+\left(p_{A}^{L}\left(x_{i}\right)-p_{B}^{L}\left(x_{i}\right)\right)^{r}+ \\ \left(v_{A}^{L}\left(x_{i}\right)-v_{B}^{L}\left(x_{i}\right)\right)^{r}+\left(u_{A}^{U}\left(x_{i}\right)-u_{B}^{U}\left(x_{i}\right)\right)^{r}+ \\ \left(p_{A}^{U}\left(x_{i}\right)-p_{B}^{U}\left(x_{i}\right)\right)^{r}+\left(v_{A}^{U}\left(x_{i}\right)-v_{B}^{U}\left(x_{i}\right)\right)^{r}\end{array}\right\}\right|^{1 / r}$

2.2.6 The normalized Geometric distance measure

$\left.d_{n r}(A, B)=\frac{1}{4} \sum_{j=1}^{4} \frac{1}{6} \sum_{i=1}^{6} \sqrt{\left\{\begin{array}{l}\left(u_{A}^{L}\left(x_{i}\right)-u_{B}^{L}\left(x_{i}\right)\right)^{2}+\left(p_{A}^{L}\left(x_{i}\right)-p_{B}^{L}\left(x_{i}\right)\right)^{2}+ \\ \left(v_{A}^{L}\left(x_{i}\right)-v_{B}^{L}\left(x_{i}\right)\right)^{2}+\left(u_{A}^{U}\left(x_{i}\right)-u_{B}^{U}\left(x_{i}\right)\right)^{2}+ \\ \left(p_{A}^{U}\left(x_{i}\right)-p_{B}^{U}\left(x_{i}\right)\right)^{2}+\left(v_{A}^{U}\left(x_{i}\right)-v_{B}^{U}\left(x_{i}\right)\right)^{2}\end{array}\right.}\right\}$

2.2.7 The Hausdorff distance measure

$$
d_{q}(A, B)=\frac{1}{4} \sum_{j=1}^{4} \frac{1}{3} \sum_{i=1}^{3} \max \left\{\begin{array}{l}
\left|u_{A}^{L}\left(x_{i}\right)-u_{B}^{L}\left(x_{i}\right)\right|,\left|u_{A}^{U}\left(x_{i}\right)-u_{B}^{U}\left(x_{i}\right)\right|+ \\
\left|v_{A}^{L}\left(x_{i}\right)-v_{B}^{L}\left(x_{i}\right)\right|,\left|v_{A}^{U}\left(x_{i}\right)-v_{B}^{U}\left(x_{i}\right)\right|+ \\
\left|p_{A}^{L}\left(x_{i}\right)-p_{B}^{L}\left(x_{i}\right)\right|,\left|p_{A}^{U}\left(x_{i}\right)-p_{B}^{U}\left(x_{i}\right)\right|
\end{array}\right\}
$$

\section{CONVERSION OF INTUITIONISTIC FUZZY SETS INTO INTERVAL-VALUED INTUITIONISTIC FUZZY SETS}

Step 1: Identify the values of membership, non-membership and hesitation of the IFS,

Step 2: The degree of membership function of IVIFS are calculated using the following formulae

$$
\begin{aligned}
& \mu_{A L}(x)=\mu_{A}-\alpha_{A} \pi_{A}(x) \\
& \mu_{A u}(x)=\mu_{A}-\alpha_{A} \pi_{A}(x)
\end{aligned}
$$

Step 3: The degree of non-membership function of IVIFS are calculated using the following formulae

$$
\begin{aligned}
& v_{A L}(x)=v_{A}-\beta_{A} \pi_{A}(x) \\
& v_{A u}(x)=v_{A}-\beta_{A} \pi_{A}(x)
\end{aligned}
$$

Step 4:Finally the IVIFS is arranged as follows

$$
A=\left\{\left\langle x,\left[\mu_{A L}(x), \mu_{A L}(x)\right],\left[v_{A L}(x), v_{A U}(x)\right], \pi_{A}(x)\right\rangle \mid x \in X\right\}
$$

\section{NUMERICAL EXAMPLES}

Let consider two IFSs $\mathrm{A}$ and $\mathrm{B}$ in $\mathrm{X}$ as follows:

$A=\{\langle a, 0.5,0.2,0.3\rangle,\langle b, 0.6,0.2,0.2\rangle,<c, 0.9,0.0,0.1\rangle\}$

4.1 Hamming Distance Measure:

$$
\begin{aligned}
& d_{H}(A, B)=\frac{1}{6} \sum_{i=1}^{n}\left\{\begin{array}{l}
\left|u_{A}\left(x_{i}\right)-u_{B}\left(x_{i}\right)\right|+ \\
\left|v_{A}\left(x_{i}\right)-v_{B}\left(x_{i}\right)\right|+
\end{array}\right\} \\
& d_{H}(A, B)=\frac{1}{6} \sum_{i=1}^{2}\{|0.2|+|0.2|+|0.2|+|-0.4|+|-0.2|+|-0.2|\}
\end{aligned}
$$

$$
d_{H}(A, B)=0.2333
$$

4.2 Normalized Hamming Distance Measure:

$$
\begin{aligned}
& d_{n H}(A, B)=\frac{1}{6 n} \sum_{i=1}^{n}\left\{\begin{array}{l}
\left|u_{A}\left(x_{i}\right)-u_{B}\left(x_{i}\right)\right|+ \\
\left|v_{A}\left(x_{i}\right)-v_{B}\left(x_{i}\right)\right|+
\end{array}\right\} \\
& d_{n H}(A, B)=\frac{1}{6 \times 2} \sum_{i=1}^{2}\{|0.2|+|0.2|+|0.2|+|-0.4|+|-0.2|+|-0.2|\} \\
& d_{n H}(A, B)=0.1167
\end{aligned}
$$

4.3 Euclidean Distance Measure:

$d_{E}(A, B)=\sqrt{\frac{1}{6} \sum_{i=1}^{n}\left\{\begin{array}{l}\left(u_{A}\left(x_{i}\right)-u_{B}\left(x_{i}\right)\right)^{2}+ \\ \left(v_{A}\left(x_{i}\right)-v_{B}\left(x_{i}\right)\right)^{2}\end{array}\right\}}$

$d_{E}(A, B)=\sqrt{\frac{1}{6}\left\{(0.5-0.3)^{2}+(0.6-0.4)^{2}+(0.9-0.7)^{2}+(0.2-0.6\}+(0.2-04)^{2}+(0.0-0.2)^{2}\right\}}$ $d_{E}(A, B)=0.2449$

4.4 Normalized Euclidean Distance Measure:

$$
\begin{aligned}
& d_{n E}(A, B)=\sqrt{\frac{1}{6 n} \sum_{i=1}^{n}\left\{\begin{array}{l}
\left(u_{A}\left(x_{i}\right)-u_{B}\left(x_{i}\right)\right)^{2}+ \\
\left(v_{A}\left(x_{i}\right)-v_{B}\left(x_{i}\right)\right)^{2}
\end{array}\right\}} \\
& d_{n E}(A, B)=\sqrt{\frac{1}{6 \times 2}\left\{(0.5-0.3)^{2}+(0.6-0.4)^{2}+(0.9-0.7)^{2}+(0.2-0.6\}+(0.2-04)^{2}+(0.0-0.2)^{2}\right\}} \\
& d_{n E}(A, B)=0.1732
\end{aligned}
$$

4.5 Geometric Distance Measure:

$$
\begin{aligned}
& d_{G}(A, B)=\sum_{i=1}^{n}\left\{\left(u_{A}\left(x_{i}\right)-u_{B}\left(x_{i}\right)\right)^{3}+\left(v_{A}\left(x_{i}\right)-v_{B}\left(x_{i}\right)\right)^{3}\right\}^{1 / 3} \\
& d_{G}(A, B)=\left((0.2)^{3}+(0.2)^{3}+(0.2)^{3}+(-0.4)^{3}+(-0.2)^{3}+(-0.2)^{3}\right)^{1 / 3} \\
& d_{G}(A, B)=0.3826
\end{aligned}
$$

4.6 Normalized Geometric Distance Measure:

$d_{n G}(A, B)=\frac{1}{2} \sum_{j=1}^{m} \frac{1}{6} \sum_{i=1}^{n}\left\{\left(u_{A}\left(x_{i}\right)-u_{B}\left(x_{i}\right)\right)^{3}+\left(v_{A}\left(x_{i}\right)-v_{B}\left(x_{i}\right)\right)^{3}\right\}^{1 / 3}$

$d_{n G}(A, B)=\frac{1}{2} \sum_{j=1}^{2} \frac{1}{6}\left((0.2)^{3}+(0.2)^{3}+(0.2)^{3}+(-0.4)^{3}+(-0.2)^{3}+(-0.2)^{3}\right)^{1 / 3}$

$d_{n G}(A, B)=0.0319$

4.7 Hausdroff's Distance Measure:

$$
\begin{aligned}
& d_{q}(A, B)=\frac{1}{2} \sum_{j=1}^{2} \frac{1}{3} \sum_{i=1}^{3} \max \left\{\begin{array}{l}
\left|u_{A}\left(x_{i}\right)-u_{B}\left(x_{i}\right)\right|,+ \\
\left|v_{A}\left(x_{i}\right)-v_{B}\left(x_{i}\right)\right|
\end{array}\right\} \\
& d_{q}(A, B)=\frac{1}{2} \sum_{j=1}^{2} \frac{1}{2}(|0.2|+|0.2|+|0.2|+|-0.4|+|-0.2|+|-0.2|) \\
& d_{q}(A, B)=0.2333
\end{aligned}
$$

The converted IVIFS:

$A=\{<a,[0.35,0.65],[0.05,0.35], 0.3>,<b,[0.5,0.7],[0.1,0.3], 0.2><c,[0.85,0.95],[0.05,0.05], 0.1>\}$ $B=\{<a,[0.25,0.35],[0.55,0.65], 0.1>,<b,[0.3,0.5],[0.3,0.5], 0.2><c,[0.65,0.75],[0.15,0.25], 0.1>\}$

4.1 Hamming Distance Measure:

$$
d_{H}(A, B)=\frac{1}{6} \sum_{i=1}^{n}\left\{\begin{array}{l}
\left|u_{A L}\left(x_{i}\right)-u_{B L}\left(x_{i}\right)\right|+\left|p_{A L}\left(x_{i}\right)-p_{B L}\left(x_{i}\right)\right|+ \\
\left|v_{A L}\left(x_{i}\right)-v_{B L}\left(x_{i}\right)\right|+\left|u_{A U}\left(x_{i}\right)-u_{B U}\left(x_{i}\right)\right|+ \\
\left|p_{A U}\left(x_{i}\right)-p_{B U}\left(x_{i}\right)\right|+\left|v_{A U}\left(x_{i}\right)-v_{B U}\left(x_{i}\right)\right|
\end{array}\right\}
$$


$d_{H}(A, B)=\frac{1}{6} \sum_{i=1}^{2}\{|0.1|+|0.3|+|-0.5|+|-0.3|+|0.2|+|0.2|+|-0.2|+|-0.2|+|0.2|+|0.2|+|-0.1|+|-0.2|\}$

\section{$d_{H}(A, B)=0.45$}

4.2 Normalized Hamming Distance Measure:

$d_{n H}(A, B)=\frac{1}{6 n} \sum_{i=1}^{n}\left\{\begin{array}{l}\left|u_{A L}\left(x_{i}\right)-u_{B L}\left(x_{i}\right)\right|+\left|p_{A L}\left(x_{i}\right)-p_{B L}\left(x_{i}\right)\right|+ \\ \left|v_{A L}\left(x_{i}\right)-v_{B L}\left(x_{i}\right)\right|+\left|u_{A U}\left(x_{i}\right)-u_{B U}\left(x_{i}\right)\right|+ \\ \left|p_{A U}\left(x_{i}\right)-p_{B U}\left(x_{i}\right)\right|+\left|v_{A U}\left(x_{i}\right)-v_{B U}\left(x_{i}\right)\right|\end{array}\right\}$

$d_{n H}(A, B)=\frac{1}{6 \times 2} \sum_{i=1}^{2}\{0.1|+| 0.3|+|-0.5|+|-0.4+|0.4+| 0.4+|-0.4+|-0.4+|0.4+| 0.4+|-0.1+|-0$. . $\}$

\section{$d_{n H}(A, B)=0.225$}

4.3 Euclidean Distance Measure:

$d_{E}(A, B)=\sqrt{\begin{array}{l}\frac{1}{6}\left\{(0.35-0.25)^{2}+(0.65-0.35)^{2}+(0.05-0.55)^{2}+\right. \\ (0.35-0.65)^{2}+(0.5-0.3)^{2}+(0.7-0.5)^{2}+ \\ (0.1-0.3)^{2}+(0.3-0.5)^{2}+(0.85-0.65)^{2}+ \\ \left.(0.95-0.75)^{2}+(0.05-0.15)^{2}+(0.05-0.25)^{2}\right\}\end{array}}$

$d_{E}(A, B)=\sqrt{\frac{1}{6}\left[0.1^{2}+0.3^{2}+0.2^{2}+0.2^{2}+0.2^{2}+0.2^{2}+0.5^{2}+0.3^{2}+0.2^{2}+0.2^{2}+0.1^{2}+0.2^{2}\right]}$

\section{$d_{E}(A, B)=0.591$}

4.4 Normalized Euclidean Distance Measure:

$\left.d_{n E}(A, B)=\sqrt{\frac{1}{6 n} \sum_{i=1}^{n}\left\{\begin{array}{l}\left(u_{A L}\left(x_{i}\right)-u_{B L}\left(x_{i}\right)\right)^{2}+\left(\pi_{A L}\left(x_{i}\right)-\pi_{B L}\left(x_{i}\right)\right)^{2}+ \\ \left(v_{A L}\left(x_{i}\right)-v_{B L}\left(x_{i}\right)\right)^{2}+\left(u_{A U}\left(x_{i}\right)-u_{B U}\left(x_{i}\right)\right)^{2}+ \\ \left(\pi_{A U}\left(x_{i}\right)-\pi_{B U}\left(x_{i}\right)\right)^{2}+\left(v_{A U}\left(x_{i}\right)-v_{B U}\left(x_{i}\right)\right)^{2}\end{array}\right.}\right\}$

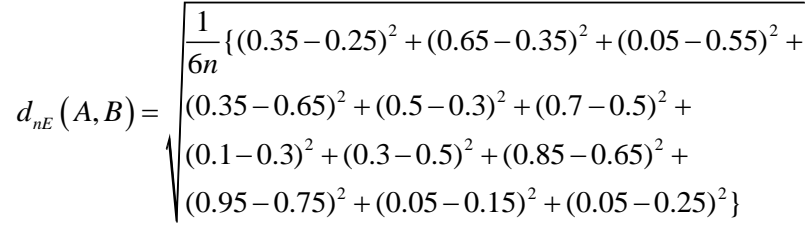

$d_{n E}(A, B)=\sqrt{\frac{1}{6 \times 2}\left[0.1^{2}+0.3^{2}+0.2^{2}+0.2^{2}+0.2^{2}+0.2^{2}+0.5^{2}+0.3^{2}+0.2^{2}+0.2^{2}+0.1^{2}+0.2^{2}\right]}$

\section{$d_{n E}(A, B)=0.2466$}

4.5 Geometric Distance Measure:

$d_{G}(A, B)=\sum_{i=1}^{n}\left\{\begin{array}{l}\left(u_{A L}\left(x_{i}\right)-u_{B L}\left(x_{i}\right)\right)^{3}+\left(v_{A L}\left(x_{i}\right)-v_{B L}\left(x_{i}\right)\right)^{3} \\ +\left(\pi_{A L}\left(x_{i}\right)-\pi_{B L}\left(x_{i}\right)\right)^{3}+\left(u_{A U}\left(x_{i}\right)-u_{B U}\left(x_{i}\right)\right)^{3} \\ +\left(v_{A U}\left(x_{i}\right)-v_{B U}\left(x_{i}\right)\right)^{3}+\left(\pi_{A U}\left(x_{i}\right)-\pi_{B U}\left(x_{i}\right)\right)^{3}\end{array}\right\}$

$d_{G}(A, B)=\left\{(0.35-0.25)^{3}+(0.65-0.35)^{3}+(0.05-0.55)^{3}\right.$

$+(0.35-0.65)^{3}+(0.5-0.3)^{3}+(0.7-0.5)^{3}+(0.1-0.3)^{3}+$

$(0.3-0.5)^{3}+(0.85-0.65)^{3}+(0.95-0.75)^{3}+(0.05-0.15)^{3}$

$\left.+(0.05-0.25)^{3}\right\}^{1 / 3}$

\section{$d_{G}(A, B)=0.4891$}

4.6 Normalized Geometric Distance Measure:

$d_{n G}(A, B)=\frac{1}{2} \sum_{j=1}^{m} \frac{1}{6} \sum_{i=1}^{n}\left\{\begin{array}{l}\left(u_{A L}\left(x_{i}\right)-u_{B L}\left(x_{i}\right)\right)^{r}+\left(v_{A L}\left(x_{i}\right)-v_{B L}\left(x_{i}\right)\right)^{r} \\ +\left(\pi_{A L}\left(x_{i}\right)-\pi_{B L}\left(x_{i}\right)\right)^{r}+\left(u_{A U}\left(x_{i}\right)-u_{B U}\left(x_{i}\right)\right)^{r} \\ +\left(v_{A U}\left(x_{i}\right)-v_{B V}\left(x_{i}\right)\right)^{r}+\left(\pi_{A U}\left(x_{i}\right)-\pi_{B U}\left(x_{i}\right)\right)^{r}\end{array}\right\}^{\frac{1}{r}}$ $d_{n G}(A, B)=\frac{1}{2} \sum_{j=1}^{2} \frac{1}{6}\left\{(0.35-0.25)^{3}+(0.65-0.35)^{3}+(0.05-0.55)^{3}\right.$

$+(0.35-0.65)^{3}+(0.5-0.3)^{3}+(0.7-0.5)^{3}+(0.1-0.3)^{3}+$

$(0.3-0.5)^{3}+(0.85-0.65)^{3}+(0.95-0.75)^{3}+(0.05-0.15)^{3}$

$\left.+(0.05-0.25)^{3}\right\}^{1 / 3}$

\section{$d_{n G}(A, B)=0.0408$}

4.7 Hausdroff's Distance Measure:

$d_{q}(A, B)=\frac{1}{2} \sum_{j=1}^{2} \frac{1}{3} \sum_{i=1}^{3} \max \left\{\begin{array}{l}\left|u_{A L}\left(x_{i}\right)-u_{B L}\left(x_{i}\right)\right|,\left|u_{A U}\left(x_{i}\right)-u_{B U}\left(x_{i}\right)\right|+ \\ \left|v_{A L}\left(x_{i}\right)-v_{B L}\left(x_{i}\right)\right|,\left|v_{A U}\left(x_{i}\right)-v_{B U}\left(x_{i}\right)\right|+ \\ \left|p_{A L}\left(x_{i}\right)-p_{B L}\left(x_{i}\right)\right|,\left|p_{A U}\left(x_{i}\right)-p_{B U}\left(x_{i}\right)\right|\end{array}\right\}$

$d_{q}(A, B)=\frac{1}{2} \sum_{I=1}^{2} \frac{1}{3}(|0.1|+|0.3|+|-0.5|+|-0.3|+|0.2|+|0.2|+|-0.2|+|-0.2|+|0.2|+|0.2|+|-0.1|+|-0.2|)$

\section{$d_{q}(A, B)=0.45$}

The distance measure in IFS and IVIFS are listed in the table shown below:

4.8 The table format of $\mathrm{A}$ and $\mathrm{B}$ according to various distance measure

\begin{tabular}{|l|c|c|}
\hline \multicolumn{1}{|c|}{ Distance Measures } & IFS & IVIFS \\
\hline Hamming Distance Measure & 0.2333 & 0.4500 \\
\hline Normalized Hamming Distance Measure & 0.1167 & 0.2250 \\
\hline Euclidean Distance Measure & 0.2449 & 0.3317 \\
\hline Normalized Euclidean Distance Measure & 0.1732 & 0.2466 \\
\hline Geometric Distance Measure & 0.3826 & 0.4891 \\
\hline Normalized Geometric Distance Measure & 0.0319 & 0.0480 \\
\hline Hausdroff's Distance Measure & 0.2333 & 0.6750 \\
\hline
\end{tabular}

4.9 Pictorial Representation of the above mentioned various distance measures both in IFS and IVIFS
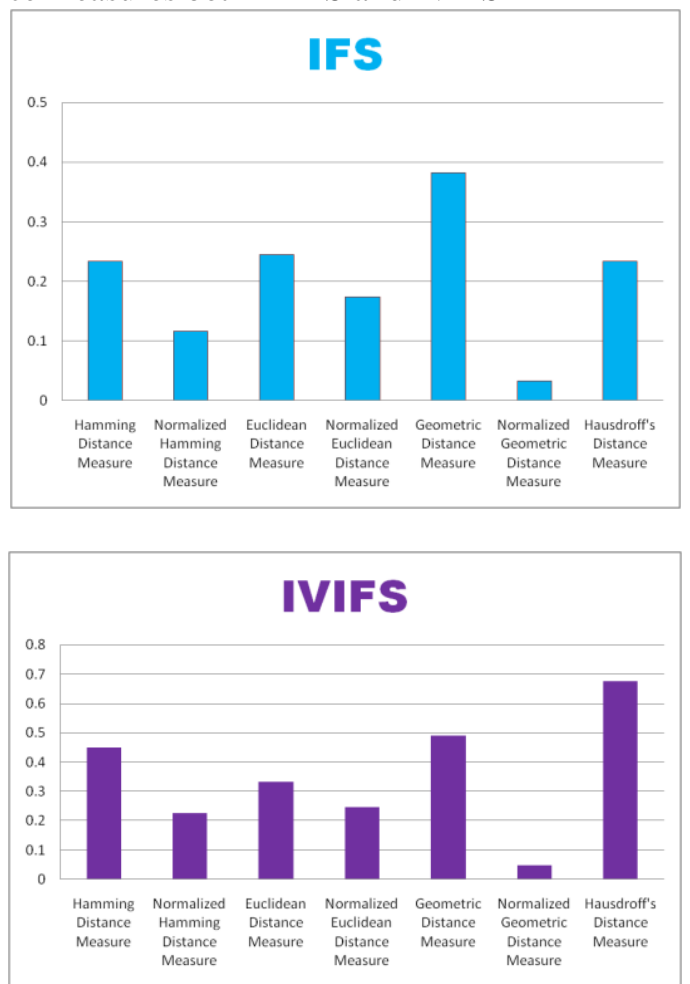

4.10 Comparative Pictorial Representation between IFS and IVIFS based on above mentioned various distance measures. 


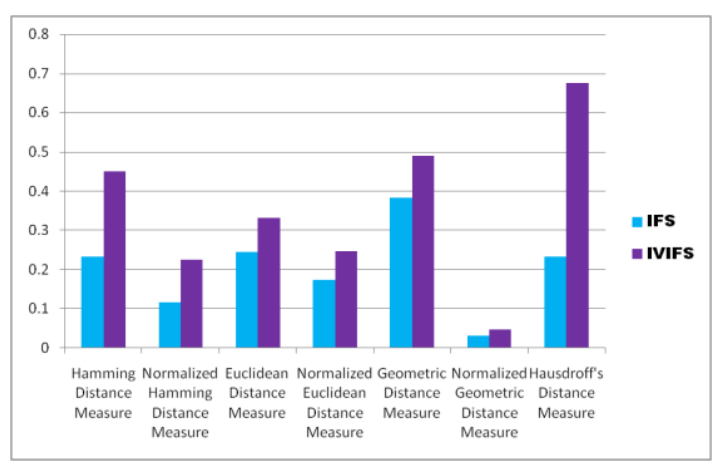

\section{CONCLUSIONS}

Here distance measure is used to find the distance between two fuzzy sets namely A and B both in IFS and IVIFS. These difference in distance is listed in the table and also their pictorial representations are indicated separately. On analyzing the Comparative Pictorial Representation between IFS and IVIFS based on above mentioned various distance measures we conclude that Hausdroff distance measure gives the highest distance between A and B but the distance differs a lot between IFS and IVIFS.

Next to the Hausdroff distance measure Geometric distance measure gives the next highest distance between $\mathrm{A}$ and $\mathrm{B}$ and also there is only slight difference in distance between IFS and IVIFS. We have ranked the distance measure based on Comparative Pictorial Representation between IFS and IVIFS.

Since in Geometric distance measure there is only slight difference in distance between IFS and IVIFS and also it gives highest distance between $\mathrm{A}$ and $\mathrm{B}$ we conclude that Geometric distance measure is one of the best distance measure. We also conclude that the difference in distance between IFS and IVIFS is due to the boundary points in IVIFS.

\section{REFERENCES}

1. R. W. Euclidean, Error detecting and error correcting codes, Bell System Technical Journal, 29 (1950) 148-161.

2. W. Huang, Y. Sh, S. Zhang, Y. Zhu, The communication complexity of the Euclidean distance problem, Information Processing Letters, 99 (2006) 149-153.

3. T. Morie, S. Matsuura, S. Miyata, T. Yamanak, M. Nagata, A. Iwata Quantum-dot structures measuring Euclidean distance for associative memories, Superlattices and Microstructure, 27 (2000) 613-616.

4. J. Gabarro-Arpa, R. Revilla, Clustering of a molecular dynamics trajectory with a Euclidean distance. Computers and Chemistry, 24 (2000) 693-698.

5. H. E. Matthew, V. P. Sergei, E. R. Paolo, Genetic code, Euclidean distance and stochastic matrices, Bulletin of Mathematical Biology, 66 (2004) 1405-1421.

6. L. Longcheng, Y Enyu, Inverse min-max spanning tree problem under the weighted sum-type Euclidean distance, Theoretical Computer Science, 396 (2008) 28-34.

7. S. Jeffrey, Euclidean distances for conjugates, Discrete Mathematics, 309 (2009) 4197-4189.

8. PUNNIAKRISHNAN, K., \& KADAMBAVANAM, K. ON INTUITIONISTIC FUZZY INVENTORY MODELS WITHOUT ALLOWING STORAGE CONSTRAINT.

9. Z. Z. Zhao, A relation between the average Euclidean distance and the average Euclidean weight of binary codes, Journal of Statistical Planning and Inference, 94 (2001) 413-419.
10. Sudha, A. S., \& Ruby, V. ANALYSIS OF MAJOR AIR POLLUTANT OF AN INDUSTRIALAREA IN COIMBATORE CITY USING FUZZY TOPSIS

11. J. Yiwei, L. Longcheng, W. Biao, Y. Enyu, Inverse minimum cost flow problems under the weighted Euclidean distance, European Journal of Operational Research, 207 (2010) 50-54.

12. YAGANOGLU, M. REAL-TIME HOME SECURITY USING RASPBERRY PI.

13. M. Izadikhah, Using the Euclidean distance to extend TOPSIS in a fuzzy environment, Journal of Computational and Applied Mathematics, 231 (2009) 200-207.

14. A. Bookstein, S. T. Klein, T. Raita, Fuzzy Euclidean distance: a new dissimilarity measure, Lecture Notes in Computer Sciences, 2089 (2006) 86-97.

15. L. A. Zadeh, Fuzzy sets, Information and Control, 8 (1965) 338-353.

16. Roseline, S. S., \& Amirtharaj, E. H. (2013). A new ranking of intuitionistic fuzzy numbers with distance method based on the circumcenter of centroids. International Journal of Applied Mathematics \& Statistical Sciences (IJAMSS), 2(4), 37-44.

17. Y. Binyamin, T. Imran, A. Lazim, W. Fatah, A new similarity measure on Intuitionistic fuzzy sets, International Journal of Computational and Mathematical Sciences, 5 (2011) 70-74.

18. K. Atanassov, Intuitionisitic Fuzzy Sets: Theory and Applications, Springer Physica-Verlag, New York, 1983.

19. Vidhya, D., Roja, E., \& Uma, M. K. (2014). Algebraic Fuzzy Roguh Sheaf Group Formed by Pointed Fuzzy Rough Topological Group. Int. J. Math. and Comp. Appl. Research, 4(1), 51-58.

20. K. Atanassov, G. Gargo, Interval-valued Intuitionistic fuzzy sets, Fuzzy Sets Systems, 31 (1989) 343-349.

21. M. M. Jose, C. Montserrat, Decision-making with distance measures and induced aggregation operators, Computer and Industrial Engineering, 60 (2011) 66-76.

22. MAHESWARI, A., \& KUMARI, P. INTERVAL-VALUED INTUITIONISTIC HESITANT FUZZY EINSTEIN GEOMETRIC AGGREGATION OPERATORS

23. M. Alibeigi, E. Ghasem Khani, T. Hajjari Islamic Azad University , A Theoretical Development on Fuzzy Distance Measure,Journal of Mathematical Extension . 3, (2015), 15-38.

24. Alblowi, S. A., Salama, A. A., \& Eisa, M. (2014). New concepts of neutrosophic sets. Infinite Study. 